\title{
ON AVERAGES OF NEWTONIAN POTENTIALS
}

\author{
MAXWELL O. READE
}

1. Introduction. Averages (mean-values) have proved extremely useful in the investigation of properties of potential functions $[\mathbf{2}, \mathbf{3}$, $4,7,9,11] ;$ to a great extent this has been due to the fact that averages of potential functions are themselves smoother potential functions.

It is the purpose of this note to exhibit the relations between the mass distribution $\sigma(E)$ associated with the potential function $A[U(x, y)]$, which is an average of a potential function $U(x, y)$, and the mass distribution $\mu(E)$ associated with $U(x, y)$. In a general sense it is proved that $\sigma(E)=A[\mu(E)]$ and that the density $D \sigma(x, y)$ of $\sigma(E)$ is the corresponding average of the density $D \mu(x, y)$. Precise statements of these results are contained in $\$ 4$ below.

It should be noted that Thompson has investigated the problem noted above [11]; except for an error in the statement of his most general result (given without proof), his results are substantially those contained here. However, whereas Thompson's method depends upon a discussion of the interchange of the order of integration in iterated Radon-Stieltjes integrals, the method of this paper depends upon the use of approximations to potentials by means of smoother potentials. Both for the sake of completeness and to point up the difference of the two methods, a proof of Thompson's (corrected) general result (which is Theorem 3 of this note), based upon Thompson's own method, is given in $\$ 4$ below.

2. Notation and definitions. Let $F$ be a closed bounded set in the $x, y$-plane, and let $\mu(e)$ be an arbitrary distribution of positive mass on $F$, that is, $\mu(e)$ is defined for all Borel sets $e$ in $F$ such that (i) $\mu(e) \geqq 0$, (ii) $\mu\left(\sum_{i=1}^{\infty} e_{i}\right)=\sum_{i=1}^{\infty} \mu\left(e_{i}\right)$, for each sequence $\left\{e_{i}\right\}$ of mutually disjoint Borel sets contained in $F[6$, p. 25]. The distribution $\mu(e)$ is said to be of finite total amount if $\mu(e)$ is uniformly bounded for all $e$ in $F$.

A distribution $\mu(e)$ may be extended so as to be defined for all Borel sets $E$ in the plane by means of the definition [3, p. 227] $\mu(E) \equiv \mu(E \cdot F)$. It is apparent that $\mu(E)$ satisfies (i) and (ii) above. In this paper it is assumed that all distributions have been thus extended to all Borel sets in the plane, although, strictly speaking, each distribution had

Presented to the Society, April 27, 1946; received by the editors March 19, 1946.

1 Numbers in brackets refer to the bibliography at the end of the paper. 
been originally defined only for those Borel sets contained in a closed bounded set $F$.

The density of $\mu(E)$ at $(x, y)$ is defined as [10, p. 149]

$$
D_{s} \mu(x, y) \equiv \lim _{\rho \rightarrow 0} \frac{\mu\left(D_{\rho}\right)}{\pi \rho^{2}}
$$

when the limit exists, where $D_{\rho}$ is the closed circular disc with center at $(x, y)$ and radius $\rho$. For distributions of the type used in this paper, it is well known that $D_{s} \mu(x, y)$ exists almost everywhere $[10$, pp. 115 , 149].

The Borel set $E$ is said to be $\mu$-regular if and only if $\mu(\bar{E}-E)=0$, where $\bar{E}$ is the point set closure of $E[5$, p. 9].

The sequence of mass distributions $\left\{\mu_{n}(E)\right\}$ is said to converge to the mass distribution $\mu(E)$ if and only if $\mu(E)=\lim _{n \rightarrow \infty} \mu_{n}(E)$ for each open $\mu$-regular set $E$. The sequence $\left\{\mu_{n}(E)\right\}$ is said to be of uniform finite total amount if and only if $\mu_{n}(E)$ is uniformly bounded for all $E$ and for all $n$.

The Newtonian potential at $(x, y)$ of the distribution $\mu(E)$ is defined by the Stieltjes-Radon integral [10, pp. 65-67]

$$
u(x, y) \equiv \iint_{W} \log \frac{1}{P Q} d \mu(\xi, \eta),
$$

where $P \equiv(x, y), Q \equiv(\xi, \eta), P Q \equiv\left((x-\xi)^{2}+(y-\eta)^{2}\right)^{1 / 2}$, and where the integral is extended over the whole finite plane $W$. It should be noted that the integral in (2) is in fact a finite integral since $\mu(E)$ vanishes for all $E$ disjoint with some closed bounded set $F$; however, the use of $W$ simplifies some of the discussion below.

$R$ will always denote an open oriented rectangle with sides parallel to the coordinate axes, while $\Re$ will always denote the open rectangle with vertices $( \pm h, \pm k)$.

If $E$ is a previously assigned Borel set, then $E_{x, y}$, for fixed $(x, y)$, denotes the set of all points $\left\{\left(x+x^{\prime}, y+y^{\prime}\right)\right\}$ for which $\left(x^{\prime}, y^{\prime}\right)$ is in $E$, and $E_{x, y}^{* *}$ denotes the set of all points $\left\{\left(x-x^{\prime}, y-y^{\prime}\right)\right\}$ for which $\left(x^{\prime}, y^{\prime}\right)$ is in $E$.

The average of $U(x, y)$ over rectangles $\Re_{x, y}$ is defined by

$$
\begin{aligned}
A_{h, k}(x, y) & \equiv A_{h, k}[U(x+\xi, y+\eta)] \\
& \equiv \frac{1}{4 h k} \iint_{\Re} U(x+\xi, y+\eta) d \xi d \eta \\
& \equiv \frac{1}{4 h k} \iint_{\Re_{x, y}} U(\xi, \eta) d \xi d \eta,
\end{aligned}
$$


while the average of $U(x, y)$ over open circular discs $D(x, y ; \rho)$, with center at $(x, y)$ and radius $\rho$, is defined by $[1,2]$

$$
\mathfrak{A}_{\rho}^{(1)}(x, y) \equiv \mathfrak{A}_{\rho}^{(1)}[U(x+\xi, y+\eta)] \equiv \frac{1}{\pi \rho^{2}} \iint_{D(x, y ; \rho)} U(\xi, \eta) d \xi d \eta,
$$

In this paper, an iterated circular average $\mathfrak{P}_{\rho}^{(3)}(x, y)[3, \mathrm{p} .236]$ will be used, where

$$
\begin{aligned}
& \mathfrak{A}_{\rho}^{(2)}(x, y) \equiv \mathfrak{A}_{\rho}^{(1)}\left[\mathfrak{A}_{\rho}^{(1)}(x+\xi, y+\eta)\right], \\
& \mathfrak{A}_{\rho}^{(3)}(x, y) \equiv \mathfrak{A}_{\rho}^{(1)}\left[\mathfrak{A}_{\rho}^{(2)}(x+\xi, y+\eta)\right] .
\end{aligned}
$$

3. Lemmas. Some of the important known results in potential theory that will be used here are listed in the form of lemmas. However, the corollary to Lemma 2, Lemma 3, and the remark following Lemma 3 are new.

Let $U(x, y)$ be a Newtonian potential with associated mass distribution $\mu(E)$, of finite total amount; then the following lemmas hold.

LEMMA 1 [3, p. 236]. $\mathfrak{A}_{\rho}(x, y) \equiv \mathfrak{U}_{\rho}^{(3)}(x, y)$ is a Newtonian potential with continuous partial derivatives of the second order, with mass distribution

$$
\mu_{\rho}(E) \equiv \iint_{E} \frac{\Delta \mathfrak{H}_{\rho}(x, y)}{2 \pi} d x d y,
$$

where $\Delta$ is the Laplace operator, such that $\mathfrak{A}_{\rho}(x, y) \nearrow U(x, y)$ as $\rho \rightarrow 0$; moreover, if $\rho$ is bounded, then $\left\{\mu_{\rho}(E)\right\}$ is of uniform finite total amount.

LEMMA $2\left[9\right.$, p. 351]. There exists a sequence $\left\{\rho_{n}\right\} \searrow 0$ as $n \rightarrow \infty$, such that $\left\{\mu_{\rho_{n}}(E)\right\}$ converges to $\mu(E)$.

Corollary. If $V(x, y)$ is another Newtonian potential with associated mass distribution $\nu(E)$, of finite total amount, then there exists a sequence $\left\{\rho_{n}\right\} \searrow 0$, as $n \rightarrow \infty$, such that $\left\{\mu_{\rho_{n}}(E)\right\}$ and $\left\{\nu_{\rho_{n}}(E)\right\}$ converge to $\mu(E)$ and $\nu(E)$, respectively; here $\nu_{\rho_{n}}(E)$ is defined by an expression analogous to (3).

Proof. The construction of the convergent sequence $\left\{\mu_{\rho_{n}}(E)\right\}$ in Lemma 2, as given by Riesz [9, pp. 351-352], follows a Cantor "diagonal" process which may be extended so as to yield a convergent sequence $\left\{\nu_{\rho_{n}}(E)\right\}$.

LEMMA 3. If $\left\{\mu_{\rho_{n}}(E)\right\}$ is the convergent sequence noted in Lemma 2, and if $R$ is a fixed rectangle in the plane, then $R_{x, y}$ is $\mu$-regular for almost all points $(x, y)$ in the plane; hence, except for a set of superficial measure 
zero it follows that

$$
\lim _{n \rightarrow \infty} \mu_{\rho_{n}}\left(R_{x, y}\right)=\mu\left(R_{x, y}\right) .
$$

Proof. Since $\mu(E)$ is essentially distributed on a closed bounded set $F$, there is a sufficiently large rectangle $R^{*}$ containing all the mass, that is, there is a rectangle $R^{*}$ containing $F$ such that $\mu(F)=\mu\left(R^{*}\right)$ $<\infty$. Now according to Reichelderfer and Ringenberg $[8$, p. 235] there are at most a countable number of values of $x,\left\{\xi_{i}\right\}$, and $y$, $\left\{\eta_{i}\right\}$, such that the mass on the lines $x=\xi_{i}$ and $y=\eta_{i}, i=1,2, \cdots$, is positive; hence, since $R$ has fixed dimensions, it follows that the boundary of $R_{x, y}$ may have positive mass if and only if the point $(x, y)$ lies on a certain plane set consisting of a countable number of lines, that is, $R_{x, y}$ is $\mu$-regular except possibly for a plane set of measure zero. The second part of the lemma follows from the definition of convergence of $\left\{\mu_{\rho_{n}}(E)\right\}$ to $\mu(E)$.

Remark. The key to the preceding proof is the result due to Reichelderfer and Ringenberg quoted above. However, that result can be extended to oblique lines as well as to other sufficiently smooth curves, and thus yield more general results. For example, under the hypothesis that $\mu(F)<\infty$, there are at most a countable number of lines with a fixed direction, say $\alpha$, that have positive mass; hence $R_{x, y}$ in (4) may be replaced by $\Pi_{x, y}$ where $\Pi$ is a certain fixed polygon.

LEMMA 4 [3, p. 231]. The limit of a monotone increasing convergent sequence of Newtonian potentials, whose mass distributions are uniformly of finite total amount, is a Newtonian potential with an associated mass distribution of finite total amount.

4. Main results. The proof of Theorem 1 illustrates the general method used in the paper.

THEOREM 1. If $U(x, y)$ is a Newtonian potential with associated mass distribution $\mu(E)$, of finite total amount, then $A(x, y) \equiv A_{h, k}(x, y)$ is a Newtonian potential with an associated mass distribution $\sigma(E)$ of finite total amount; moreover, $\sigma(E)$ is unique and given by each of the representations

$$
\sigma(E)=\iint_{E} \frac{\mu\left(\Re_{x, y}\right)}{4 h k} d x d y,
$$

and

$$
\sigma(E)=\frac{1}{4 h k} \iint_{\Re} \mu\left(E_{x, y}\right) d x d y .
$$


Proof. Since $U(x, y)$ satisfies the hypothesis of Lemma 1, it follows that there is a sequence $\left\{\rho_{n}\right\} \searrow 0$, as $n \rightarrow \infty$, such that $\left\{\mu_{\rho_{n}}(E)\right\}$ converges to $\mu(E)$, where $\mu_{\rho_{n}}(E)$ is defined by (3). Moreover, by Lemma $1, \mathfrak{2}_{\rho_{n}}^{(3)}(x, y) \equiv \mathfrak{A}_{n}(x, y)$ is sufficiently smooth so that Fubini's classic theorem may be used to prove that

$$
\begin{aligned}
\mathfrak{A}_{n} A(x, y) & \equiv \mathfrak{A}_{\rho_{n}}^{(3)}\left[A_{h, k}(x+\xi, y+\eta)\right]=A_{h, k}\left[\mathfrak{A}_{\rho_{n}}^{(3)}(x+\xi, y+\eta)\right] \\
& \equiv A \mathfrak{A}_{n}(x, y)
\end{aligned}
$$

holds. But $\mathfrak{A}_{n}(x, y)$ is a Newtonian potential, so that (7) yields

(8) $\mathfrak{A}_{n} A(x, y)=\frac{1}{4 h k} \iint_{\mathfrak{R}}\left[\iint_{W} \log \frac{1}{P Q} \frac{\Delta \mathfrak{A}_{n}(s, t)}{2 \pi} d s d t\right] d \xi d \eta$,

where $P \equiv(x+\xi, y+\eta)$ and $Q \equiv(s, t)$. If $(s, t)$ is replaced by $(s+\xi, t+\eta)$ then $(8)$ becomes

$$
\mathfrak{A}_{n} A(x, y)
$$

$$
=\frac{1}{4 h k} \iint_{\mathfrak{R}}\left[\iint_{W} \log \frac{1}{P^{\prime} Q} \frac{\Delta \mathscr{A}_{n}(s+\xi, t+\eta)}{2 \pi} d s d t\right] d \xi d \eta,
$$

where $P^{\prime} \equiv(x, y)$. If Fubini's theorem is applied to (9), then

$$
\begin{aligned}
& \mathfrak{A}_{n} A(x, y) \\
& \quad=\iint_{W} \log \frac{1}{P^{\prime} Q}\left[\frac{1}{4 h k} \iint_{\Re} \frac{\Delta \mathfrak{H}_{n}(s+\xi, t+\eta)}{2 \pi} d \xi d \eta\right] d s d t
\end{aligned}
$$

holds; now an application of both Leibnitz' rule and Fubini's theorem to $(10)$ yields

$$
\mathfrak{A}_{n} A(x, y)=\iint_{W} \log \frac{1}{P^{\prime} Q} \frac{\Delta \mathfrak{A}_{n} A(s, t)}{2 \pi} d s d t .
$$

From (11) it follows that $\mathfrak{A}_{n} A(x, y)=A \mathfrak{A}_{n}(x, y)$ is a Newtonian potential with mass distribution

$$
\sigma_{\rho_{n}}(E) \equiv \iint_{E} \frac{\Delta \mathfrak{A}_{n} A(x, y)}{2 \pi} d x d y .
$$

By Lemma $1, \mathfrak{A}_{n}(x, y) \nearrow U(x, y)$ as $n \rightarrow \infty$; hence (7) yields $\mathfrak{A}_{n} A(x, y) \nearrow A(x, y)$, as $n \rightarrow \infty$. Therefore, by Lemmas 1 and 4 , $A(x, y)$ is a Newtonian potential with an associated mass distribution (of finite total amount), say $\sigma(E)$.

By the corollary to Lemma 2, there is a sequence $\left\{\rho_{n}^{\prime}\right\} \searrow 0$ as $n \rightarrow \infty$, such that $\left\{\mu_{\rho_{n}^{\prime}}^{\prime}(E)\right\}$ converges to $\mu(E)$ and $\left\{\sigma_{\rho_{n}^{\prime}}^{\prime}(E)\right\}$ converges to $\sigma(E)$, 
where $\sigma_{\rho_{n}^{\prime}}(E)$ is defined by an expression similar to (3). If the substitutions $\mathfrak{Y}_{\rho_{n}^{\prime}}^{(3)}(x, y) \equiv a_{n}(x, y), \mathfrak{H}_{\rho_{n}^{\prime}}^{(3)} A(x, y) \equiv a_{n} A(x, y), \mu_{\rho_{n}^{\prime}}(E) \equiv \mu_{n}(E)$, and $\sigma_{\rho_{n}^{\prime}}(E) \equiv \sigma_{n}(E)$ are made, then it follows that for each $R$,

$$
\mu_{n}(R) \equiv \iint_{R} \frac{\Delta a_{n}(x, y)}{2 \pi} d x d y
$$

and

$$
\sigma_{n}(R) \equiv \iint_{R} \frac{\Delta a_{n} A(x, y)}{2 \pi} d x d y
$$

must hold.

Now (13) may be written in the forms

$$
\begin{aligned}
\sigma_{n}(R) & =\iint_{R}\left[\frac{1}{4 h k} \iint_{\Re} \frac{\Delta a_{n}(x+\xi, y+\eta)}{2 \pi} d \xi d \eta\right] d x d y, \\
& =\iint_{R} \frac{\mu_{n}\left(\Re_{x, y}\right)}{4 h k} d x d y
\end{aligned}
$$

and

$$
\begin{aligned}
\sigma_{n}(R) & =\frac{1}{4 h k} \iint_{\Re}\left[\iint_{R} \frac{\Delta a_{n}(x+\xi, y+\eta)}{2 \pi} d x d y\right] d \xi d \eta, \\
& =\frac{1}{4 h k} \iint_{\Re} \mu_{n}\left(R_{\xi, \eta}\right) d \xi d \eta .
\end{aligned}
$$

Since $\left\{\sigma_{n}(E)\right\}$ converges to $\sigma(E)$, it follows from (14) and Lemma 3 that

$$
\sigma\left(R^{*}\right)=\lim _{n \rightarrow \infty} \sigma_{n}\left(R^{*}\right)=\iint_{R^{*}} \frac{\mu\left(\Re_{x, y}\right)}{4 h k} d x d y
$$

holds for all $\sigma$-regular $R^{*}$. Since each rectangle $R$ is the point set limit of a monotone increasing sequence of $\sigma$-regular rectangles, say $\left\{R_{n}^{*}\right\} \nearrow R$, such that $\left[8\right.$, p. 236] $\lim _{n \rightarrow \infty} \sigma\left(R_{n}^{*}\right)=\sigma(R)$, it follows from (16) that

$$
\sigma(R)=\iint_{R} \frac{\mu\left(\Re_{x, y}\right)}{4 h k}
$$

holds for all rectangles $R$.

Let $D$ be any large open disc that contains $F$ in the interior, such that the distance between the boundaries of $F$ and $D$ exceeds $(h+k)$. The mass distribution defined by 


$$
\sigma^{*}(E) \equiv \iint_{E} \frac{\mu\left(\Re_{x, y}\right)}{4 h k} d x d y
$$

is an extension of the rectangle function (17) to the class of mass distributions within $D$; moreover, $\sigma(E)$ is such an extension of (17) too. But Reichelderfer and Ringenberg have shown that the extension of a rectangle function such as (17) to the class of mass distributions defined for all Borel sets within $D$ is unique $[8, \mathrm{p} .234]$, that is, $\sigma(E)=\sigma^{*}(E)$ for all Borel sets $E$ within $D$. Since $D$ was an arbitrary, large disc, it follows that $\sigma(E)=\sigma^{*}(E)$ for all Borel sets in the plane. Hence (5) holds.

In a similar way, (6) may be derived from (15) and the "extension theorem" of Reichelderfer and Ringenberg noted above. This completes the proof.

COROLlaRy 1. Under the hypotheses of Theorem 1, the relation

$$
D_{s} \sigma(x, y)=\frac{\mu\left(\Re_{x, y}\right)}{4 h k}
$$

holds almost everywhere.

Proor. A proof follows at once from (1) and (5).

In keeping with the remark made following Lemma 3, one can state the following theorem and corollary whose proofs would follow the lines of the preceding two proofs.

Theorem 2. Let $T$ be a fixed figure in the plane, of superficial measure $|T| \neq 0$, and let $A(x, y)$ be the average of the Newtonian potential $U(x, y)$, defined in Theorem 1, over $T_{x, y}$. If the analogue of Lemma 3 holds, that is, if $\lim _{n \rightarrow \infty} \mu_{\rho_{n}}\left(T_{x, y}\right)=\mu\left(T_{x, y}\right)$, almost everywhere, then $A(x, y)$ is a Newtonian potential with associated mass distribution

$$
\sigma(E)=\iint_{E} \frac{\mu\left(T_{x, y}\right)}{|T|} d x d y=\frac{1}{|T|} \iint_{T} \mu\left(E_{x, y}\right) d x d y,
$$

of finite total amount.

COROLlaRY 2. Under the hypotheses of Theorem 2,

$$
D_{s} \sigma(x, y)=\frac{\mu\left(T_{x, y}\right)}{|T|}
$$

holds almost everywhere.

It is now a simple matter to obtain the following general result. 
THEOREM 3. Let $G$ be a fixed open set in the plane, with superficial measure $|G|<\infty$, and let $A(G ; x, y)$ be the average of the Newtonian potential $U(x, y)$, defined in Theorem 1 , over $G_{x, y}$. Then $A(G ; x, y)$ is a Newtonian potential with associated mass distribution

$$
\sigma(E)=\iint_{E} \frac{\mu\left(G_{x, y}\right)}{|G|} d x d y=\frac{1}{|G|} \iint_{G} \mu\left(E_{x, y}\right) d x d y,
$$

of finite total amount.

Proof. Since $G$ is an open set in the plane, $|G|<\infty$, there exists a monotone increasing sequence of open sets $\left\{T_{n}\right\}$, such that each $T_{n}$ is the sum of a finite number of simple, open polygons, and such that $\left(T_{n}\right)_{x, y} \nearrow G_{x, y}$, as $n \rightarrow \infty$, for each $x, y$. In what follows, $T$ will denote a member of the sequence $\left\{T_{n}\right\}$, and instead of using the symbol " $n \rightarrow \infty$," the symbol " $T \nearrow A$ " will be used.

It is necessary to approximate the average

$$
\begin{aligned}
A(G ; x, y) & \equiv \frac{1}{|G|} \iint_{G} U(x+\xi, y+\eta) d \xi d \eta \\
& =\frac{1}{|G|} \iint_{G_{x, y}} U(\xi, \eta) d \xi d \eta
\end{aligned}
$$

One such approximation is the average,

$$
\begin{aligned}
A(T ; x, y) & \equiv \frac{1}{|T|} \iint_{T} U(x+\xi, y+\eta) d \xi d \eta \\
& =\frac{1}{|T|} \iint_{T_{x, y}} U(\xi, \eta) d \xi d \eta
\end{aligned}
$$

Now by virtue of the remark following Lemma 3, it follows that Theorem 2 applies to the average (21) because $T$ is the sum of a finite number of simple polygons. Hence $A(T ; x, y)$ is a Newtonian potential of the form

$$
A(T ; x, y)=\iint_{W} \log \frac{1}{P Q} \frac{\mu\left(T_{\xi, \eta}\right)}{|T|} d \xi d \eta,
$$

where $P \equiv(x, y), Q \equiv(\xi, \eta)$, and where (18) holds. Moreover, since $\mu(E)$ is a distribution of positive mass, it follows that

$$
\mu\left(T_{\xi, \eta}\right) \nearrow \mu\left(G_{\xi, \eta}\right), \quad \text { as } T \nearrow A,
$$

holds for all $\xi, \eta$. Now it follows from (20), (21), (22), and (23) that 


$$
A(G ; x, y)=\lim _{T \nearrow_{A}} A(T ; x, y)=\iint_{W} \log \frac{1}{P Q} \frac{\mu\left(G_{\xi, \eta}\right)}{|G|} d \xi d \eta
$$

holds for all $(x, y)$. It now follows from (24) and the definition of a Newtonian potential that $A(G ; x, y)$ is a Newtonian potential having a summable density $D \sigma(x, y)$ for which

$$
D \sigma(x, y)=\frac{\mu\left(G_{x, y}\right)}{|G|}
$$

holds almost everywhere.

The relation (19) now follows from (18) and (25). This completes the proof.

Another proof of the preceding theorem will now be given; this proof is based upon Thompson's method [11].

From (2) and (20) we have

(26) $A(G ; x, y) \equiv A(x, y)=\frac{1}{|G|} \iint_{G_{x, y}} d \xi d \eta \iint_{W} \log \frac{1}{M Q} d \mu(s, t)$,

where $M \equiv(s, t), Q \equiv(\xi, \eta)$. Since the integrand in (26) is a lower semicontinuous function, the order of integration may be interchanged; hence

$$
A(x, y)=\frac{1}{|G|} \iint_{W} d \mu(s, t) \iint_{G x, y} \log \frac{1}{M Q} d \xi d \eta
$$

But

$$
\iint_{G_{x, \nu}} \log \frac{1}{M Q} d \xi d \eta=\iint_{G_{z, \ell}^{* *}} \log \frac{1}{P Q} d \xi d \eta
$$

hence (27) becomes

$$
\begin{aligned}
A(x, y) & =\frac{1}{|G|} \iint_{W} d \mu(s, t) \iint_{G_{s, t}^{* *}} \log \frac{1}{P Q} d \xi d \eta \\
& =\frac{1}{|G|} \iint_{W} d \mu(s, t) \iint_{W} \log \frac{1}{P Q} d \nu_{s, t}(\xi, \eta),
\end{aligned}
$$

where

$$
\nu_{s, t}(E) \equiv\left|E \cdot G_{s, t}^{* *}\right| \text {. }
$$

Now it follows from a fundamental result of Thompson [11] that (28) may be written in the form 


$$
A(x, y)=\frac{1}{|G|} \iint_{W} \log \frac{1}{P Q} d\left[\iint_{W} \nu_{s, t}(\xi, \eta) d \mu(s, t)\right] .
$$

Consider $\sigma(E)$ defined by

$$
\sigma(E)=\iint_{W} \nu_{\varepsilon, t}(E) d \mu(s, t) .
$$

From (29) and (31) it follows that

$$
\begin{aligned}
\sigma(E) & =\iint_{W}\left|E \cdot G_{s, t}^{* *}\right| d \mu(s, t) \\
& =\iint_{W} d \mu(s, t) \iint_{E \cdot G_{z ;}, t} d u d v \\
& =\iint_{W} d \mu(s, t) \iint_{W} B(u, v ; s, t) d u d v \\
& =\iint_{W} d u d v \iint_{W} B(u, v ; s, t) d \mu(s, t),
\end{aligned}
$$

where

$$
\begin{aligned}
B(u, v ; s, t) & =1, & (u, v) \text { in } E \cdot G_{s, t}^{* *}, \\
B(u, v ; s, t) & =0, & (u, v) \operatorname{not} \text { in } E \cdot G_{s, t .}^{* *}
\end{aligned}
$$

But for each $(u, v)$ in $E, B(u, v ; s, t)$ vanishes except for $(s, t)$ in $G_{u, v}$. Hence (33) yields the first part of (19).

Now (19), (29), (30), and (32) yield (24), which is a Newtonian potential with density (25); this latter holds almost everywhere.

Since $\mu(F)<\infty$ and $F$ is bounded, it follows from (31) that $\sigma(E)$ is bounded. This completes the proof.

At this point it should be remarked that Thompson's error consisted of writing $G_{x, y}^{* *}$ for $G_{x, y}$ in (19) and (25).

5. Conclusion. Additional results may be obtained by considering point averages and averages over curves.

It should be noted that the following interesting result follows from (19):

$$
\iint_{E} \mu\left(G_{x, y}\right) d x d y=\iint_{G} \mu\left(E_{x, y}\right) d x d y
$$

for each open set $G$ and for each set $E$ measurable Borel. It would be 
interesting to see a direct proof of this equality based upon Fubini's theorem.

Added in proof. Professor $\mathrm{H}$. Federer has informed the author that he has found a direct proof of (34).

\section{BIBLIOGRAPHY}

1. H. E. Bray, Proof of a formula for an area, Bull. Amer. Math. Soc. vol. 29 (1923) pp. 264-270.

2. E. F. Beckenbach and Maxwell Reade, Mean-values and harmonic polynomials, Trans. Amer. Math. Soc. vol. 53 (1943) pp. 230-238.

3. G. C. Evans, On potentials of positive mass. I, Trans. Amer. Math. Soc. vol. 37 (1935) pp. 226-253.

4. - On potentials of positive mass. II, Trans. Amer. Math. Soc. vol. 38 (1935) pp. 201-236.

5. O. Frostman, Potential d'équilibre et capacité des ensembles, avec quelques applications d la theorie des fonctions, Thesis, Lund, 1935.

6. T. Rad6, Subharmonic functions, Berlin, 1937.

7. Maxwell Reade, Some remarks on subharmonic functions, Duke Math. J. vol. 10 (1943) pp. 531-536.

8. $P$. Reichelderfer and $L$. Ringenberg, The extension of rectangle functions, Duke Math. J. vol. 8 (1941) pp. 231-242.

9. F. Riesz, Sur les fonctions subharmoniques et leur rapport à la therorie du potentiel, II, Acta Math. vol. 54 (1930) pp. 321-360.

10. S. Saks, Theory of the integral, New York, 1937.

11. J. M. Thompson, Distribution of mass for averages of Newtonian potential functions, Bull. Amer. Math. Soc. vol. 41 (1935) pp. 744-752.

Purdue University 